\title{
Lipid polymeric nanoparticles modified with tight junction-modulating peptides promote afatinib delivery across a blood-brain barrier model
}

\author{
Yu-Li Lo ${ }^{1,2,3^{*}+}$ (1) , Hua-Ching Lin ${ }^{4,5 \dagger}$, Shu-Ting Hong ${ }^{1}$, Chih-Hsien Chang ${ }^{1}$, Chen-Shen Wang ${ }^{1}$ and \\ Anya Maan-Yuh Lin $2,6^{*}$
}

${ }^{*}$ Correspondence:

yulilo@nycu.edu.tw;

yulilo@ym.edu.tw;

myalin@vghtpe.gov.tw;

myalin@nycu.edu.tw

†Yu-Li Lo and Hua-Ching Lin

are co-first authors

${ }^{1}$ Institute of Pharmacology,

National Yang Ming Chiao

Tung University, Taipei 112,

Taiwan

${ }^{6}$ Department of Medical

Research, Taipei Veterans

General Hospital, Taipei 112,

Taiwan

Full list of author information is available at the end of the article

\begin{abstract}
Background: Brain metastases from non-small cell lung cancer (NSCLC) remain one of the most challenging malignancies. Afatinib (Afa) is an orally administered irreversible ErbB family blocker approved for epidermal growth factor receptor (EGFR)-mutated NSCLC. However, the incidence of brain metastases in patients with NSCLC and EGFR mutation is high. One of the major obstacles in the treatment of brain metastases is to transport drugs across the blood-brain barrier (BBB). A lipid polymeric nanoparticle ( $L P N)$ modified with a tight junction-modulating peptide is a potential formulation to deliver therapeutics across the BBB. FD7 and CCD are short peptides that perturb the tight junctions (TJs) of the BBB. In this study, the use of LPN modified with FD7 or CCD as a delivery platform was explored to enhance Afa delivery across the BBB model of mouse brain-derived endothelial bEnd.3 cells.
\end{abstract}

Results: Our findings revealed that Afa/LPN-FD7 and Afa/LPN-CCD exhibited a homogeneous shape, a uniform nano-scaled particle size, and a sustained-release profile. FD7, CCD, Afa/LPN-FD7, and Afa/LPN-CCD did not cause a significant cytotoxic effect on bEnd. 3 cells. Afa/LPN-FD7 and Afa/LPN-CCD across the bEnd. 3 cells enhanced the cytotoxicity of Afa on human lung adenocarcinoma PC9 cells. FD7 and CCD-modulated TJ proteins, such as claudin 5 and ZO-1, reduced transendothelial electrical resistance, and increased the permeability of paracellular markers across the bEnd.3 cells. Afa/LPNFD7 and Afa/LPN-CCD were also partially transported through clathrin- and caveolaemediated transcytosis, revealing the effective activation of paracellular and transcellular pathways to facilitate Afa delivery across the BBB and cytotoxicity of Afa on PC9 cells.

(c) The Author(s) 2021. This article is licensed under a Creative Commons Attribution 4.0 International License, which permits use, sharing, adaptation, distribution and reproduction in any medium or format, as long as you give appropriate credit to the original author(s) and the source, provide a link to the Creative Commons licence, and indicate if changes were made. The images or other third party material in this article are included in the article's Creative Commons licence, unless indicated otherwise in a credit line to the material. If material is not included in the article's Creative Commons licence and your intended use is not permitted by statutory regulation or exceeds the permitted use, you will need to obtain permission directly from the copyright holder. To view a copy of this licence, visit http://creativecommons.org/ licenses/by/4.0/. The Creative Commons Public Domain Dedication waiver (http://creativecommons.org/publicdomain/zero/1.0/) applies to the data made available in this article, unless otherwise stated in a credit line to the data. 
Conclusion: TJ-modulating peptide-modified LPN could be a prospective platform for the delivery of chemotherapeutics across the BBB to the brain for the potential treatment of the BM of NSCLC.

Keywords: Afatinib, Blood-brain barrier, Lipid polymeric nanoparticle, Tight junctionmodulating peptide, Lung cancer, Brain metastasis

\section{Background}

Non-small-cell lung cancer (NSCLC) is the most common lung cancer and has become a critical cause of cancer-related deaths globally (Dempke et al. 2015). Patients with NSCLC, especially Asians, are highly associated with epidermal growth factor receptor (EGFR) mutations (Fidler 2015). About 30\% of patients with NSCLC develop metastasis in the central nervous system (CNS), such as brain metastases (BM) and leptomeningeal metastases (LM), within the first 2 years after the diagnosis of primary tumors, particularly those harboring EGFR mutation (Dempke et al. 2015; Wei et al. 2019). The systemic administration of EGFR-tyrosine kinase inhibitors (TKIs) is one of the possible treatments (Abdallah and Wong 2018). However, the poor penetration of gefitinib and erlotinib, two first-generation EGFR-TKIs, across the blood-brain barrier $(\mathrm{BBB})$ remains a primary hindrance for this therapeutic regimen (Zeng et al. 2015). The BBB is crucial to regulate CNS homeostasis by maintaining the balance of material exchange between the circulation and the brain (Trabulo et al. 2010). Importantly, the neuroprotective BBB is a dynamic barrier with tight junctions (TJs) to prevent organisms and potential neurotoxins from attacking the CNS (Chen and Liu 2012). The BBB possesses special features, including low paracellular permeability, because of TJs and efflux pump proteins such as P-glycoprotein (On and Miller 2014).

Afatinib (BIBW2992; abbreviated as Afa) is an orally active and second-generation EGFR-TKI. It shows a potent and irreversible effect on suppressing pan-erythroblastic leukemia viral oncogene homolog (ErbB) family, including EGFR, human EGFR 2 (HER2), and HER4 (Hurvitz et al. 2014). Interestingly, evidence has shown the promising results of Afa as a first-line therapy in NSCLC with EGFR mutations and active CNS metastases (Li et al. 2015). Particularly, Afa circumvents resistance to third-generation TKI osimertinib in patients suffering from NSCLC with LM baring-acquired EGFR L858R/L718Q mutation (Rijcken et al. 2007). Although no significant differences are detected in intracranial treatment responses between groups treated with Afa at doses of 30 and $40 \mathrm{mg}$ (Wei et al. 2019), the incidence of rash/acne and paronychia in the group treated with $40 \mathrm{mg}$ of Afa is considerably higher than that in the group treated with $30 \mathrm{mg}$ of Afa (Sasikala et al. 2015). This result suggests the demand of low-dose Afa regimen for reducing the intolerable systemic side effects accompanied with Afa treatment. However, the dose reduction of Afa may lead to a low Afa concentration in the cerebral spinal fluid (CSF) and cause treatment failure against BM (Liu et al. 2017). Afa partially transports across the $\mathrm{BBB}$ and penetrates into the $\mathrm{BM}$ at low concentrations. Because of its low $\mathrm{IC}_{50}$ against different EGFR variants, Afa may still demonstrate clinical efficacy on limited CNS metastases (Hoffknecht et al. 2015). However, this low Afa concentration seems not enough to treat BM in NSCLC with the EGFR mutation of exon 19 
deletion, exon 20 T790M, or exon 21 L858R (Wei et al. 2019). Furthermore, the phase III randomized trial of Afa in patients of trastuzumab-resistant breast cancer with brain metastasis is stopped early through unfavorable risk-benefit analysis (Hurvitz et al. 2014). Moreover, Afa also shows the disadvantage of low solubility in water and commonly used solvents. Hence, it is pivotal to design nanoparticles with multiple functions of modulating the $\mathrm{BBB}$, targeting the cancer focus, and inducing the controlled release of Afa at the site of tumors. The successful development of nanoparticle formulations may provide a potential delivery system for enhancing anticancer efficacy and reducing the associated adverse events of Afa.

Lipid polymeric nanoparticle (LPN) is a potential formulation to deliver therapeutics into the brain by improving common difficulties, such as the BBB penetration, solubility, and toxicity (Sim et al. 2020). It comprises the polymeric core of hydrophobic polylacticco-glycolic acid (PLGA) coated with lipid layers as the shell (Mandal et al. 2016). This design combines the advantages of both lipophilic polymeric nano-core to encapsulate Afa and amphiphilic lipid-PEG shell, which can be further conjugated with antibodies or peptides for different purposes (Hong et al. 2019). Accordingly, modifying the surface properties of nanoparticles by PEGylation and peptides to disturb TJs of the BBB is a possible strategy for improving drug penetration into the brain (Masserini 2013; Trabulo et al. 2013). Previous studies showing promising results have indicated that FD7 peptide and cyclic (CD) peptide (abbreviated as CCD peptide) are two short peptides that can increase the BBB permeability and have the potential to disrupt the TJs of the BBB (Alaofi et al. 2017, 2016; Bocsik et al. 2016; Herman et al. 2007). FD7 peptide (FDFWITP) was found through screening the phage display peptide library (Herman et al. 2007). It shows the effects on TJ proteins, especially claudins. Claudins are involved in the paracellular ion flux of TJs (Herman et al. 2007). FD7 peptide may bind to the TJ complex or other associated assemblies, particularly claudin 1 or 5 (Herman et al. 2007). This 7-mer peptide enhances the ion permeability and disturbs $\mathrm{TJ}$ proteins on the BBB model (Bocsik et al. 2016; Deli 2009; Herman et al. 2007). Intriguingly, FD7 in nontoxic concentrations elicits reversible and more $\mathrm{TJ}$-modulating response in the $\mathrm{BBB}$ than in intestinal cells (Bocsik et al. 2016; Deli 2009). CCD peptide (CDTPPVC) is a cyclic derivative from the extracellular 1 (EC1) domain of human E-cadherin (Laksitorini et al. 2015). CCD modulates E-cadherin-mediated cell-cell adhesion and inhibits the adherens junctions of the vascular endothelial cells forming the BBB (Alaofi et al. 2016; Laksitorini et al. 2015). These interactions may generate pores in the intercellular junctions of the BBB and enhance paracellular drug penetration across the BBB (Alaofi et al. 2017, 2016; Laksitorini et al. 2015). CCD has been found to bind to the EC1 domain of human E-cadherin protein as verified with the NMR spectrum (Alaofi et al. 2017). In the present study, the use of Afa-loaded LPN coated with TJ-modulated peptides, such as FD7 and CCD (Afa/LPN-FD7 and Afa/LPN-CCD), as a platform was explored to enhance Afa delivery across the BBB model of mouse brain-derived endothelial cells.3 (bEnd.3) cells to inhibit the growth of human lung adenocarcinoma PC9 cells. 
Table 1 Characterization of Afa/LPN and peptide-modified LPNS

\begin{tabular}{lllll}
\hline Formulations & Particle size $(\mathbf{n m})$ & PdI $^{\mathbf{a}}$ & Zeta potential $^{(\mathbf{m V})}$ & EE\% $^{\mathbf{b}}$ \\
\hline LPN & $145.26 \pm 2.48$ & $0.20 \pm 0.04$ & $-15.28 \pm 2.16$ & - \\
Afa/LPN & $168.61 \pm 4.10$ & $0.22 \pm 0.03$ & $-13.83 \pm 0.49$ & $87.52 \pm 1.72$ \\
Afa/LPN-FD7 & $166.12 \pm 8.91$ & $0.21 \pm 0.02$ & $-10.74 \pm 1.42$ & $87.33 \pm 1.54$ \\
Afa/LPN-CCD & $150.18 \pm 0.31$ & $0.23 \pm 0.03$ & $-25.32 \pm 1.50$ & $86.83 \pm 1.62$ \\
\hline
\end{tabular}

a $P d l$ polydispersity index

${ }^{\mathrm{b}}$ EE\% encapsulation efficiency

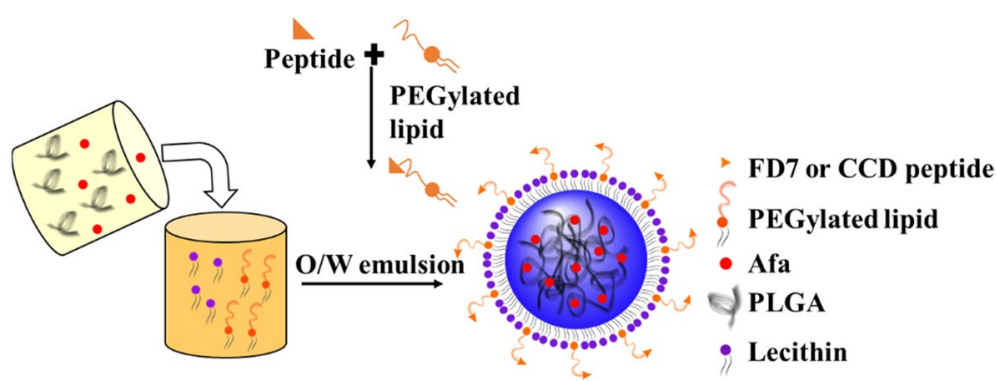

Fig. 1 Schematic of the formation of Afa-loaded and peptide-modified LPN. Peptides include FD7 or CCD peptide

\section{Results}

Characterization of Afa encaspulated in LPN (Afa/LPN) and peptife-modified LPN (Afa/ LPN-FD7 and Afa/LPN-CCD)

The physicochemical characteristics of Afa/LPN, Afa/LPN-FD7, and Afa/LPN-CCD are demonstrated in Table 1. A scheme of preparing peptide-conjugated LPNs is shown in Fig. 1. The size of all formulations was $145.26 \pm 2.48$ to $168.61 \pm 4.10 \mathrm{~nm}$ (Fig. 2A-C, Table 1). Furthermore, the particle size was evaluated for 28 days and only displayed a slight change (Additional file 1: Figure S1), indicating that the particle size remained constant for at least 28 days. The polydispersity index (PdI) was approximately 0.2 , indicating that these nanoparticle formulations were well dispersed and homogeneous (Table 1). The zeta potentials of Afa/LPN, Afa/LPNFD7, and Afa/LPN-CCD were $-13.8 \pm 0.49,-10.74 \pm 1.42$, and $-25.32 \pm 1.50 \mathrm{mV}$, respectively. All the nanoparticles were negatively charged, and the encapsulation efficiency (EE, \%) of these formulations was as high as approximately $87 \%$ (Table 1). The images of transmission electron microscopy (TEM) showed that the nanoparticles were spherical with an FD7 or CCD peptide-coating shell layer around the PLGA core in which Afa was incorporated (Fig. 2D-F).

\section{In vitro release of Afa from various $L P N$ formulations}

The in vitro release of Afa, Afa loaded in LPN, and FD7- or CCD-conjugated LPN were performed through dialysis in phosphate-buffered saline (PBS, pH 6.5; Fig. 3). This experiment was carried out using the initial Afa concentration of $10 \mu \mathrm{M}$ in different formulations. Afa-contained formulations were placed in the dialysis device and immersed in PBS at $37{ }^{\circ} \mathrm{C}$ for $48 \mathrm{~h}$. The data indicated that free Afa released $72.21 \% \pm 6.15 \%$ in the first $1 \mathrm{~h}$. However, the release percentages of Afa from Afa/LPN, Afa/LPN-FD7, 

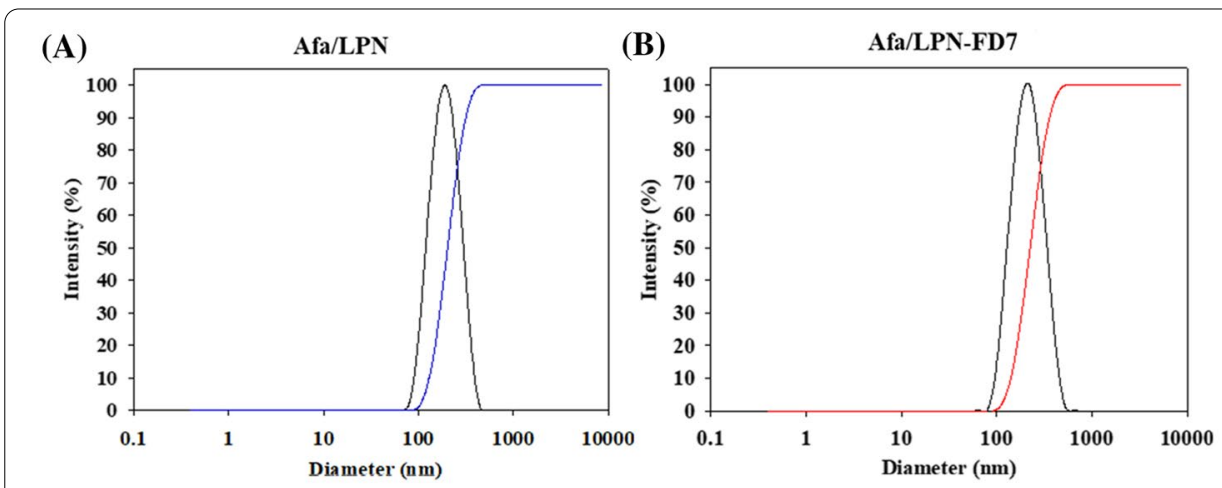

(C)

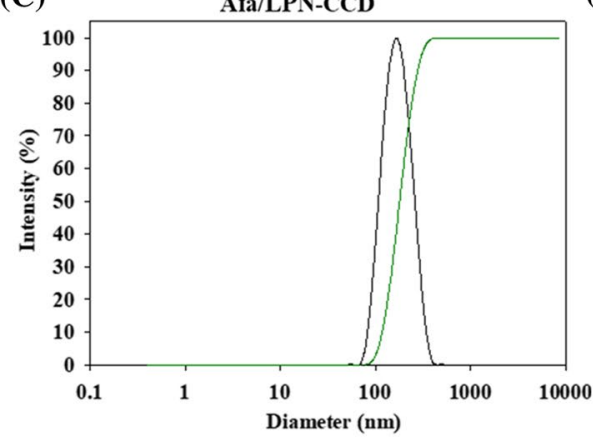

(E)

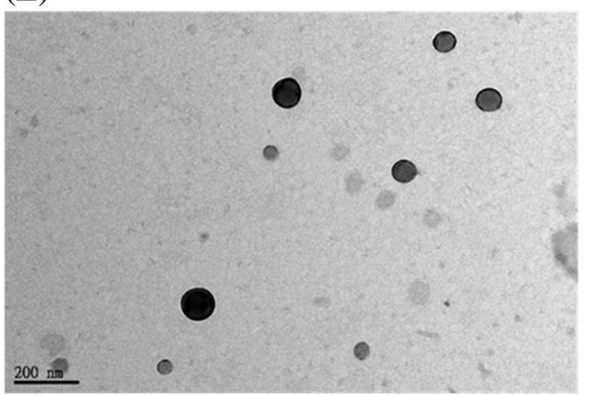

(D)

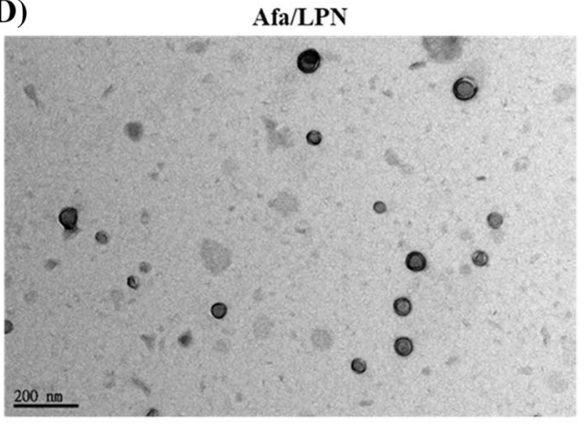

(F)

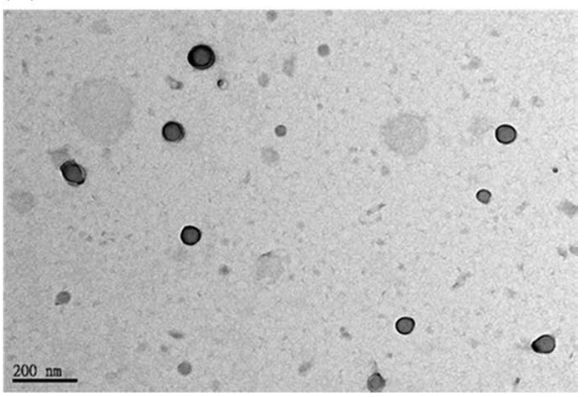

Fig. 2 Particle size distribution of A Afa/LPN, B Afa/LPN-FD7, and C Afa/LPN-CCD and transmission electron microscopic images of $\mathbf{D} A f a / L P N, \mathbf{E} A f a / L P N-F D 7$, and $\mathbf{F}$ Afa/LPN-CCD

and Afa/LPN-CCD were lower than that of Afa (Fig. 3). After $8 \mathrm{~h}, 60.26 \% \pm 2.58 \%$ and $56.18 \% \pm 1.29 \%$ of Afa were released from Afa/LPN-FD7 and Afa/LPN-CCD, respectively, which were remarkably lower than that from Afa/LPN (73.57\% $\pm 4.81 \%)$. Afa released from FD7- or CCD-conjugated LPN was below $80 \%$ for $48 \mathrm{~h}$ in a sustainedrelease manner. By contrast, free Afa released approximately $95 \%$ for $48 \mathrm{~h}$.

\section{Cytotoxicity of FD7, CCD, and Afa formulations in bEnd.3 and/or PC9 cells}

The cytotoxicity of FD7 and CCD peptides and FD7- and CCD-conjugated LPN on bEnd.3 cells was measured via a sulforhodamine B (SRB) assay (Fig. 4A, B). In Fig. 4A, $\mathrm{B}, 36 \mu \mathrm{M}$ FD7 and CCD (marked as \#) was the selected concentration for the conjugation of FD7 and CCD with LPN, and the viability of bEnd. 3 cells was maintained at 


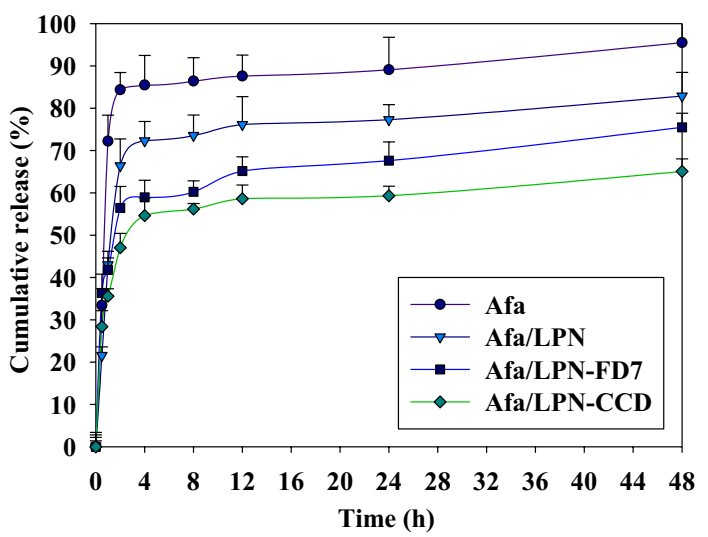

Fig. 3 In vitro release of Afa from LPN, LPN-FD7, and LPN-CCD. Afa released from various formulations was performed in the dialysis bag in PBS (pH 6.5) at $37^{\circ} \mathrm{C}$

approximately $90 \%$. Furthermore, the cytotoxicity of different concentrations of Afa and Afa/LPN was determined in bEnd. 3 and PC9 cells for $48 \mathrm{~h}$ to identify the suitable concentrations of Afa and Afa/LPN formulations with low toxicity to bEnd.3 cells, respectively (Fig. 4C-E). The viability of bEnd.3 cells decreased to around $70 \%$ when Afa concentration reached $1000 \mathrm{nM}\left(1 \mu \mathrm{M}\right.$; Fig. 4C). $\mathrm{IC}_{50}$ of Afa in PC9 cells was $1.6 \mathrm{nM}$ (Fig. 4D). Hence, $1.6 \mathrm{nM}$ Afa was used in the following experiments. Particularly, Afa (1.6 nM) did not elicit significant cytotoxic effects on bEnd.3 cells (Fig. 4C). Our results verified that $1.6 \mathrm{nM} \mathrm{Afa/LPN}$, Afa/LPN-FD7, and Afa/LPN-CCD (marked as \#) did not cause noticeable toxic effects on bEnd.3 cells (Fig. 4E). However, Afa (1.6 nM) encapsulated in LPN, LPN-FD7, and LPN-CCD significantly improved the Afa cytotoxicity on PC-9 cells (Fig. 4F).

\section{Establishment of the in vitro BBB model}

An in vitro BBB model was established by growing bEnd. 3 cells on Transwell inserts (Fig. 5A), and the representative image of the BBB model was captured with a Nikon microscope (Fig. 5B). The bEnd.3 cells were stained with SRB, and the morphological characteristics showed that the bEnd.3 monolayer was uniform (Fig. 5B). The integrity of the BBB was investigated by detecting transendothelial electrical resistance (TEER) and permeability (\%) levels. bEnd. 3 cells were seeded on the insert and cultured for 6 days. The TEER value was detected every day. After the culture of the indicated days, the TEER values increased from $10.23 \pm 2.34 \Omega \cdot \mathrm{cm}^{2}$ to $132.24 \pm 3.12 \Omega \cdot \mathrm{cm}^{2}$ and reached a plateau after 5 days of incubation (Fig. $5 \mathrm{C}$ ). Moreover, the relative transendothelial permeability percentage of fluorescein isothiocyanate-conjugated dextran (FITC-dextran; MW 70,000) across the BBB model was $8.52 \% \pm 1.16 \%$ compared with that without BBB (blank; 100\%; Fig. 5D). In previous studies, the permeability of the BBB model is also evaluated by using FITC-dextran as a paracellular marker (Li et al. 2010; Lin et al. 2016). Hence, the establishment of the BBB model was confirmed via morphological, TEER value, and permeability (\%) studies (Fig. 5B-D). 

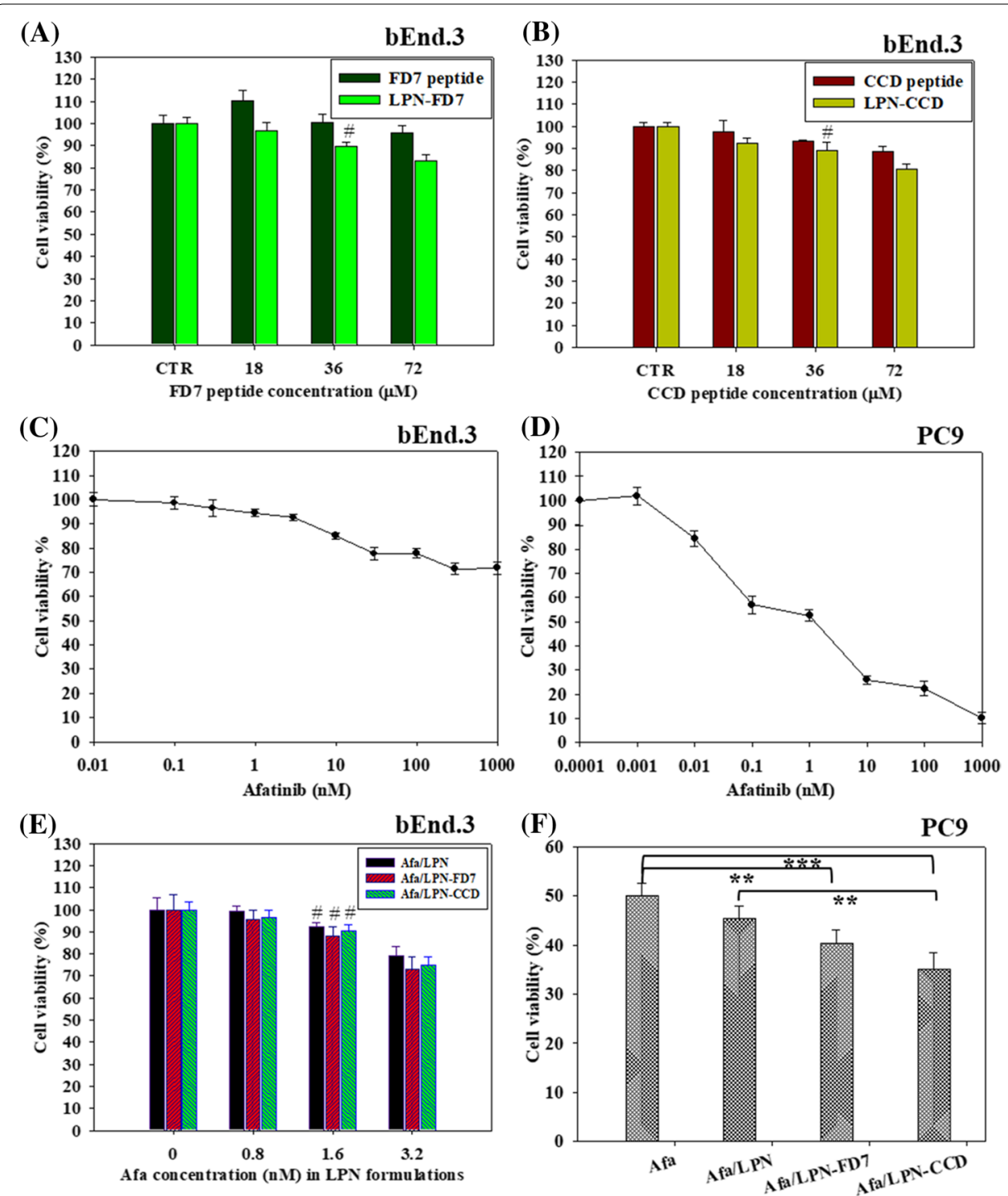

Afa (1.6 $\mathrm{nM})$ in various $L P N$ formulations

Fig. 4 Cytotoxicity of FD7, FD7-LPN, CCD, CCD-LPN, and Afa in different formulations on bEnd.3 and PC9 cells. Cytotoxicity of different concentrations of $\mathbf{A}$ FD7 and FD7-LPN, B CCD, CCD-LPN, and C Afa on bEnd.3 cells, D Afa on PC9 cells, E Afa/LPN, Afa/LPN-FD7, and Afa/LPN-CCD on bEnd.3 cells for $48 \mathrm{~h}$, and $\mathbf{F}$ Afa/LPN, Afa/LPN-FD7, and Afa/LPN-CCD on PC9 cells for $48 \mathrm{~h}$. Cell viability was measured via SRB assay. Values are the mean \pm S.D. $(n=3)$. (A, B, E) \# represents the concentrations of FD7, CCD, Afa, and Afa/LPN with low cytotoxic effects on bEnd. 3 cells, which were used in the following experiments. $\mathbf{F}^{*}$ statistical significance at $p<0.05$; ${ }^{* *} p<0.01 ; * * * 0.001$

\section{Cytotoxicity of Afa in various formulations across the BBB on PC9 cells}

The schematic (Fig. 6A) and result in Fig. 6B-D demonstrated the cytotoxicity of different Afa-formulations across the BBB model of Transwell inserts on PC9 cells in the absence or presence of bEnd. 3 cells. The cell viability of the blank group was measured when $1.6 \mathrm{nM}$ Afa was transported across the insert without bEnd.3 cells. Afa under this condition decreased the viability of PC9 cells to $66.88 \% \pm 7.65 \%$ without the BBB (Fig. 6B). However, the viability of PC9 cells after Afa treatment returned to $85.52 \% \pm 4.19 \%$ when the bEnd. 3 cells were incubated on the insert, indicating that 
(A)

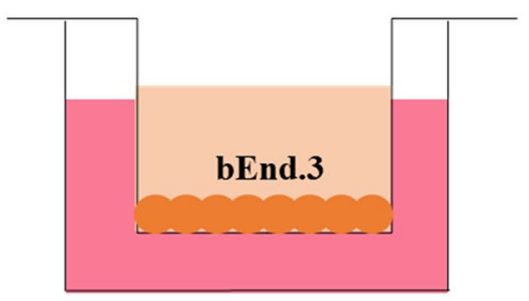

(C)

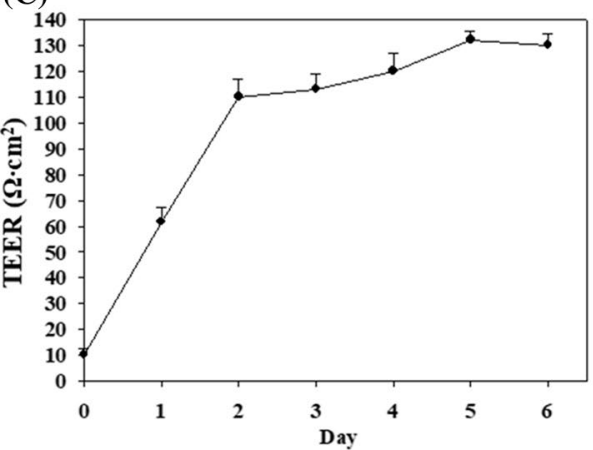

(B)

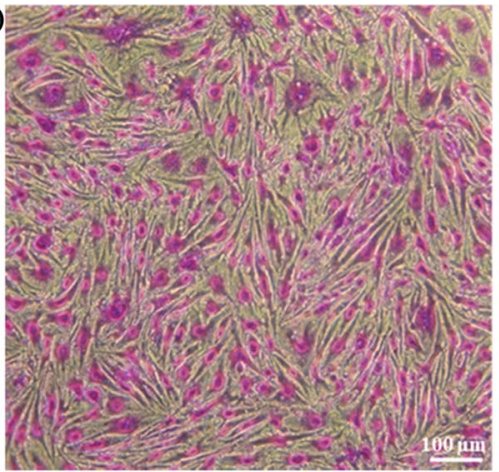

(D)

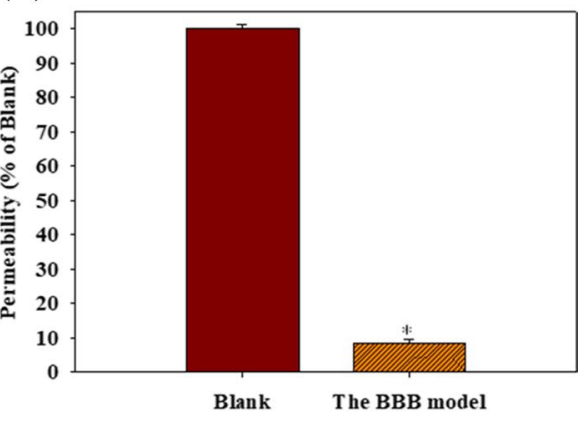

Fig. 5 Establishment of an in vitro blood-brain barrier (BBB) model. A Schematic of the in vitro BBB model; B morphological characteristics of the BBB model stained with sulforhodamine B (SRB). Representative image of an intact cell monolayer was captured under a Nikon light microscope (magnification, 100 x); C transendothelial electrical resistance (TEER) values were evaluated 6 days after seeding; $\mathbf{D}$ permeability of fluorescein isothiocyanate (FITC)-dextran (MW 70,000) across the BBB model on day 5. Values are the mean \pm S.D. $(n=3) .{ }^{*} p<0.05$ : compared with blank via Student's t-test

the $\mathrm{BBB}$ model functioned as a barrier to diminish Afa's delivery into the brain for BM treatment. Interestingly, when Afa was encapsulated in LPN, the viability of PC9 cells decreased to $65.71 \% \pm 3.36 \%$, which was similar to that of the free Afa group without the BBB. Afa-loaded LPN-FD7 and LPN-CCD across the BBB further reduced the viabilities of PC9 cells to $52.89 \% \pm 4.29 \%$ and $43.79 \% \pm 3.11 \%$, respectively (Fig. $6 \mathrm{~B}$ ). There were approximately further $33 \%$ and $42 \%$ decreases on PC9 viability for Afa/LPN-FD7 and Afa/LPN-CCD, respectively, compared to Afa (Fig. 6B), suggesting a superior effect of these two tight junction-modulating peptides on promoting Afa delivery across the $\mathrm{BBB}$ model. The cytotoxic effect of Afa/LPN-CCD was higher across the BBB on PC9 cells than that of Afa/LPN-FD7 (Fig. 6B).

Moreover, the protein expression of PC9 cells after the treatment with various Afa formulations across the bEnd. 3 cells for $48 \mathrm{~h}$ was examined through Western blot. Bcl-2, an anti-apoptotic protein, was considerably suppressed with the treatments of Afa/LPN, Afa/LPN-FD7, and Afa/LPN-CCD (Fig. 6C). By contrast, the expression levels of proapoptotic proteins, including Bax and caspase-3, were gradually escalated with the treatments of Afa/LPN, Afa/LPN-FD7, and Afa/LPN-CCD. Free Afa did not cross the insert with bEnd.3 cells very well to display its potency on inducing Bax and inhibiting Bcl-2. However, Afa in LPN, LPN-FD7, and LPN-CCD seemed to considerably cross the BBB 
(A)

(B)
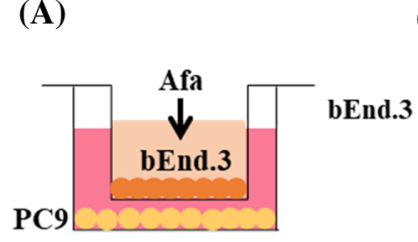

\begin{tabular}{r|c|c|c|c|c|c|} 
Insert & + & + & + & + & + & + \\
\cline { 2 - 7 } (BBB) & - & + & + & + & + & + \\
\cline { 2 - 7 } PC9 & + & + & + & + & + & + \\
\cline { 2 - 7 } & & & &
\end{tabular}
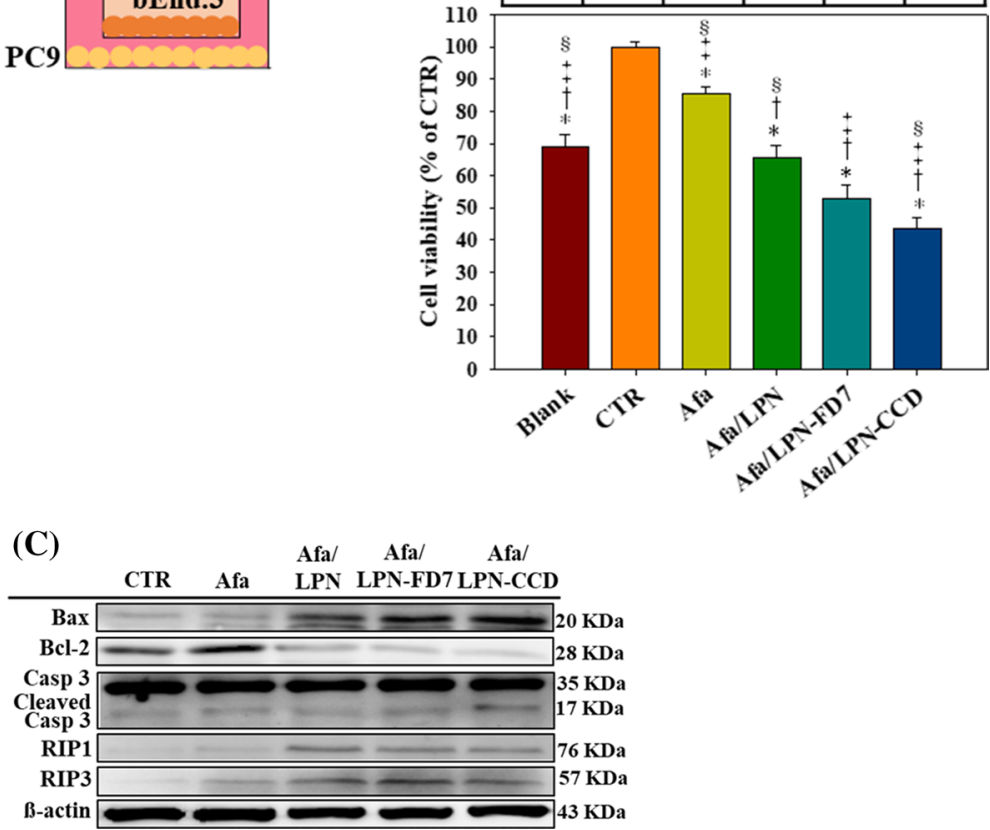

Fig. 6 Effect of Afa in various formulations on the cytotoxicity of PC9 cells and the barrier integrity of the BBB model. A Schematic of Afa across the BBB model; $B$ relative cell viability (\%) of Afa, Afa/LPN, and peptide-modified LPN across the BBB on PC9 cells. PC9 cells were treated with Afa, Afa/LPN, Afa/LPN-FD7, and Afa/LPN-CCD with or without the BBB for $48 \mathrm{~h}$. Cell viability was measured via SRB assay. Values are the mean \pm S.D. $(n=3) .{ }^{*} p<0.05$ compared with the control $(C T R),{ }^{\dagger} p<0.05$ compared with $\mathrm{Afa}^{\neq}{ }^{\ddagger} p<0.05$ compared with Afa/LPN, and ${ }^{\S} p<0.05$ compared with Afa/LPN-FD7 through Student's t-test analysis. C Protein expression levels of apoptosis, including Bax, Bcl-2, and caspase 3, and necroptosis such as RIP1 and 3 were detected with Western blot after the above treatment in PC9 cells

model via transcytosis to show the effect on triggering apoptosis (Fig. 6C). Afa encapsulated in LPN, LPN-FD7, and LPN-CCD remarkably induced Bax and suppressed Bcl-2, suggesting the good carrier capability of LPN, especially modified by CCD, on the delivery of Afa across the BBB model. Additionally, necroptosis mediators, such as kinase receptor-interacting protein 1 (RIP1) and RIP3, were upregulated by the treatments of Afa/LPN, Afa/LPN-FD7, and Afa/LPN-CCD (Fig. 6C). Therefore, these formulations elicited partial cytotoxic effects on PC9 cells via necroptosis.

\section{Cellular uptake mechanisms and barrier function of the BBB model}

FD7 and CCD are TJ-modulated peptides (Herman et al. 2007; Laksitorini et al. 2015). Figure $6 \mathrm{~B}$ shows that the cell growth inhibition of Afa/LPN-FD7 and Afa/LPN-CCD was higher across the BBB in PC9 cells than that of Afa/LPN. However, the cellular uptake mechanisms of Afa/LPN-FD7 and Afa/LPN-CCD formulations on bEnd.3 cells require further investigation. Rhodamine 123 (Rh123) was used as a fluorescent probe of Afa for encapsulation into nanoparticles. The bEnd.3 cells were pretreated with various endocytosis inhibitors, including chlorpromazine (CPZ), 5-( $N, N$-dimethyl) amiloride (DMA), 
and nystatin, for $1 \mathrm{~h}$ and then incubated with Rh123-loaded LPN-FD7 or LPN-CCD for $3 \mathrm{~h}$. The fluorescence intensity of each group with or without endocytosis inhibitors was detected through flow cytometry, and the means were normalized relative to Rh123/ LPN-FD7 (Fig. 7A) or Rh123/LPN-CCD (Fig. 7B). When the cells were pretreated with CPZ (clathrin-mediated endocytosis inhibitor) and nystatin (caveolae-mediated endocytosis inhibitor, the relative fluorescence intensity (\%) of Rh123/LPN-FD7 decreased to $67.71 \% \pm 1.63 \%$ and $80.83 \% \pm 4.46 \%$ compared with that of Rh123/LPN-FD7 without inhibitor (Fig. 7A). Similarly, the pretreatment of cells with CPZ and nystatin decreased the fluorescence intensity of Rh123/LPN-CCD to $78.29 \% \pm 3.27 \%$ and $74.23 \% \pm 12.56 \%$ compared with that of the Rh123/LPN-CCD group (Fig. 7B). Furthermore, Afa incorporated in the nanoparticles across the BBB might enhance the cytotoxic effect on PC9 cells by decreasing TEER values and increasing the permeability of the bEnd. 3 cells compared with those of the free Afa group (Fig. 7C, D). The TEER value of Afa/LPN-FD7 and Afa/LPN-CCD groups decreased to $74.50 \% \pm 3.13 \%$ and $65.14 \% \pm 2.08 \%$ (Fig. $7 \mathrm{C}$ ). Afa/LPN-FD7 and Afa/LPN-CCD increased the permeability of FITC-dextran (MW $70,000)$ across the BBB to $157.49 \% \pm 5.08 \%$ and $165.42 \pm 7.79 \%$, which significantly differed from those of the control (CTR; 100.00\% $\pm 5.54 \%$ ), Afa, and Afa/LPN groups $(p<0.05$; Fig. 7D). Accordingly, LPN-FD7 and LPN-CCD formulations crossed the BBB not only via the paracellular pathway through the opening of the TJs (Fig. $7 \mathrm{C}, \mathrm{D}$ ) but also via clathrin- and caveolae-mediated transcytosis pathways (Fig. 7A, B). Thus, the enhanced delivery of Afa in LPN-FD7 and LPN-CCD formulations across the bEnd.3 cells caused a higher degree of cytotoxic effect on PC9 cells than that of Afa or Afa/LPN (Fig. 6B). Importantly, after removal of Afa/LPN-FD7 or Afa/LPN-CCD for $3 \mathrm{~h}$, the relative transendothelial permeability percentage of FITC-dextran (MW 70,000) across the BBB model was $8.11 \% \pm 3.28 \%$ and $9.27 \% \pm 1.69 \%$ compared with that without bEnd.3 cells (blank; 100\%). Furthermore, the TEER values were $130.78 \pm 4.32$ and $131.21 \pm 3.86$ $\Omega \cdot \mathrm{cm}^{2}$ after removal of Afa/LPN-FD7 or Afa/LPN-CCD for $3 \mathrm{~h}$, suggesting a reversible opening of the in vitro BBB barrier and the recovery of integrity of the BBB model.

\section{Junctional morphology of bEnd.3 cells through confocal laser microscopy (CLSM) and junctional protein expression of bEnd. 3 cells through Western blot}

The junctional morphology of bEnd.3 cells was examined through CLSM because FD7 and CCD peptides affect TJs (Herman et al. 2007; Laksitorini et al. 2015). Zonula occludens-1 (ZO-1) and claudin-5 are the major junction proteins of the $\mathrm{BBB}$, so we observed the morphological characteristics of these proteins after the treatment of bEnd. 3 cells with Afa, Afa/LPN-FD7, and Afa/LPN-CCD for $48 \mathrm{~h}$. The CLSM images indicated that bEnd. 3 cells without treatment exhibited the continuous junctional morphology of ZO-1 and claudin-5 (Fig. 8A). The morphological characteristics of the cells treated with Afa were similar to those of the cells in the control group. Afa did not demonstrate the obvious alteration in the junction proteins of ZO-1 and claudin-5 (Fig. 8). Nevertheless, the junctions of ZO-1 and claudin- 5 were discontinuous after the cells were treated with Afa/LPN-FD7 and Afa/LPN-CCD (Fig. 8A). The junctional proteins in the Afa/LPN-CCD group were apparently disturbed (Fig. 8A). This phenomenon indicated that the nanoparticles conjugated with $\mathrm{TJ}$-modulated peptides might disrupt the arrangement of junctional proteins, such as $\mathrm{ZO}-1$ and claudin-5, consequently 

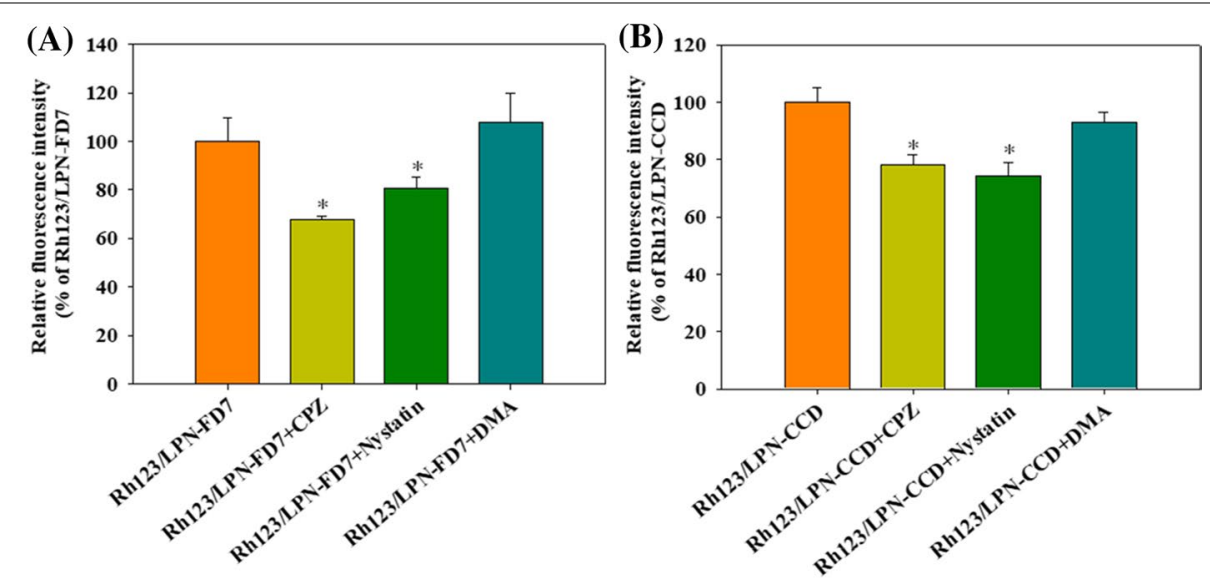

(C)

(D)
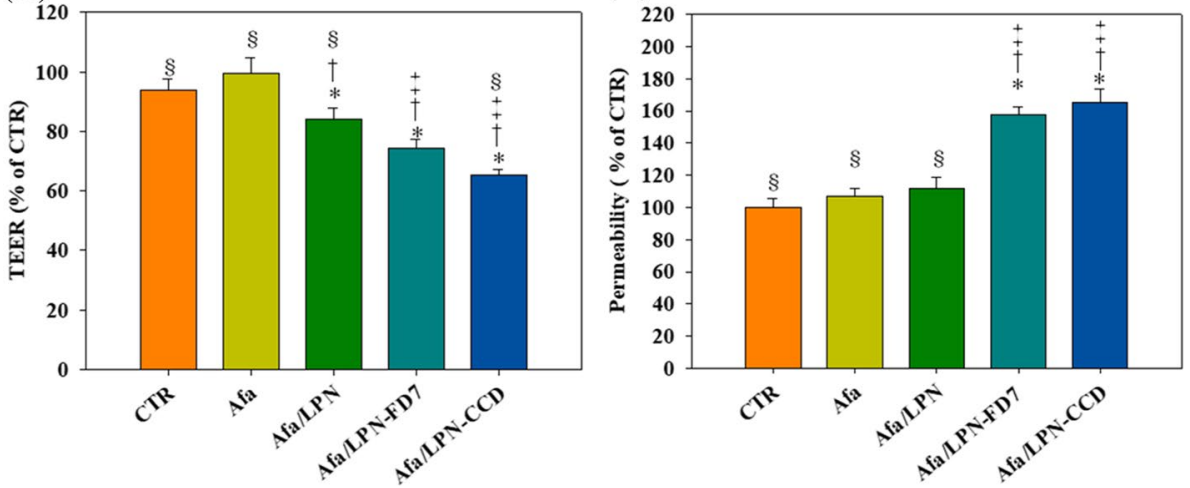

Fig. 7 Cellular uptake mechanisms and barrier function of the BBB model. A Relative fluorescence intensity (\%) of Rh123/LPN-FD7 and B Rh123/LPN-CCD after the treatment of bEnd.3 cells with different endocytosis inhibitors, including CPZ (clathrin-mediated endocytosis inhibitor), DMA (macropinocytosis inhibitor), and nystatin (caveolae-mediated endocytosis inhibitor) for $1 \mathrm{~h}$ and then incubated with Rh123-loaded LPN-FD7 or LPN-CCD for 3 h. Values are the mean \pm S.D. $(n=3)$. (A, B): ${ }^{*} p<0.05$ compared with $\mathbf{A}$ Rh123/LPN-FD7 and B Rh123/LPN-CCD through Student's t-test analysis, respectively. C TEER after $48 \mathrm{~h}$ of the treatment of various Afa formulations on bEnd.3 cells. D Permeability (\%) of FITC-dextran (MW 70,000) across the bEnd.3 cells after $48 \mathrm{~h}$ of the treatment of various Afa formulations. C, D Values are the mean \pm S.D. $(n=3)$. ${ }^{*} p<0.05$ compared with CTR, ${ }^{\dagger} p<0.05$ compared with Afa, ${ }^{\neq} p<0.05$ compared with Afa/LPN, and ${ }^{\S} p<0.05$ compared with Afa/ LPN-FD7 through Student's t-test analysis

enhancing the Afa transport across the BBB. Additionally, the junctional protein expression of bEnd. 3 cells was also evaluated after the treatment with different Afa formulations through Western blot. However, Afa/LPN-FD7 and Afa/LPN-CCD did not exhibit an obvious effect on the junctional protein expression of bEnd.3 cells, including ZO-1, claudin-5, and E-cadherin; this result suggested the perturbing effect of Afa/LPN-FD7 and $\mathrm{Afa} / \mathrm{LPN}-\mathrm{CCD}$ on the arrangement of TJ-associated proteins instead of suppressing the junctional protein levels (Fig. 8).

\section{Discussion}

Afa was approved by the FDA in 2013 as a first-line treatment for patients with EGFRspecific mutations, including exon 19 deletions or exon 21 (L858R) substitutions (Lee et al. 2014). The covalent bond between the acrylamide of Afa and the cysteine residue within the active site of the intracellular tyrosine kinase domain of EGFR, HER2, and 


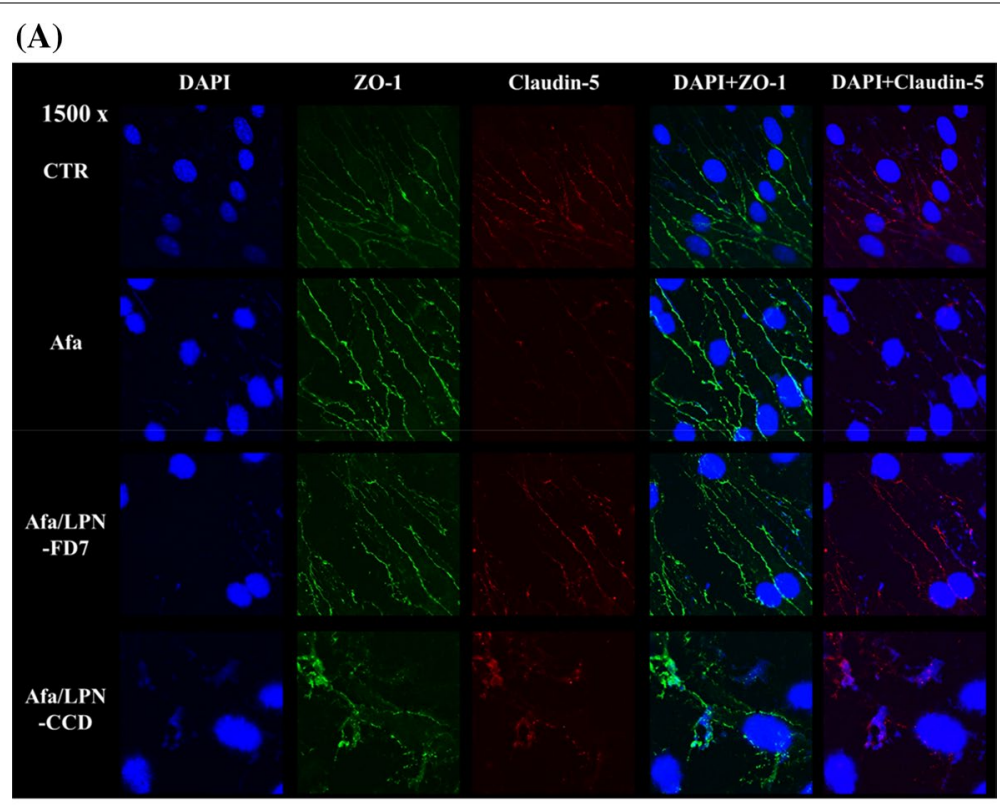

(B)

$$
\begin{array}{cccc}
\text { CTR Afa } & \text { Afa/ Afa/ Afa/ } & \text { APN LPN-FD7LPN-CCD } \\
\hline
\end{array}
$$

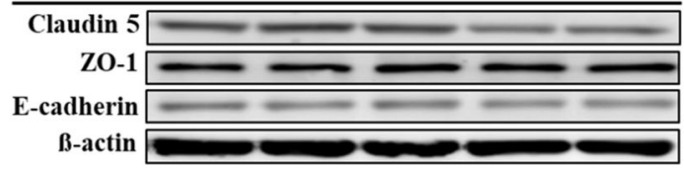

Fig. 8 Effect of peptide-modified LPN on the junctional morphology and protein expression of bEnd.3 cells. A Immunostaining of the TJ proteins ZO-1 and claudin- 5 after the treatment of bEnd. 3 cells with Afa, Afa/ LPN-FD7, and Afa/LPN-CCD for 48 h observed through confocal laser scanning microscopy (magnification, 1500x). Green: ZO-1. Red: claudin-5. Blue: the cell nuclei stained with DAPI. B Protein expression levels of claudin-5, ZO-1, and E-cadherin were detected through Western blot after the above treatment in bEnd.3 cells

HER4 caused the irreversible inhibition of downstream signaling pathways, effectively inhibited cell growth, and induced apoptosis in NSCLC (D'Arcangelo and Hirsch 2014; $\mathrm{Li}$ et al. 2008). A recent investigation has indicated that three first-line EGFR-TKIs, such as gefitinib, erlotinib, and Afa, demonstrate a comparable treatment efficacy based on the results of progression-free survival and overall survival in patients with EGFRmutated NSCLC and brain metastases (Rijcken et al. 2005). Nevertheless, limited information was reported for the effective brain concentration and the intracranial response rates of Afa (Hoffknecht et al. 2015). For the effective treatment of NSCLC with EGFR mutation, Afa at the dose of $40 \mathrm{mg}$ daily was commonly recommended by the European Medicines Agency (Yang et al. 2016). Although Afa has been generally regarded as predictable adverse events, such as cutaneous and gastrointestinal (GI) symptoms (Hirsh 2015), it causes some serious side effects, including grades 3-4 diarrhea (14\%), rash or acne (16\%), and paronychia (11\%) (Yang et al. 2015). The dose-limiting GI toxicities and the detrimental effects due to the irreversible inhibition of EGFR by Afa may halt the further clinical application of Afa at a high dose. However, the dose reduction of Afa because of the intolerable side effects accompanied with high-dose Afa often causes the low concentration of Afa in the CSF and the treatment failure against BM (Liu et al. 
2017). Interestingly, the surface modification of nanoparticles to disturb TJs of the BBB may enhance drug delivery to the brain (Masserini 2013). In the current study, two nanoparticle Afa formulations, including Afa/LPN-FD7 and Afa/LPN-CCD, were successfully developed. These two nanoformulations exhibited a homogeneous shape, acceptable particle size and surface potential, and a sustained-release profile (Table 1, Figs. 2 and 3). FD7 and CCD peptides increase the BBB permeability with the potential to interrupt TJs of the BBB (Alaofi et al. 2017; Herman et al. 2007). In a previous research, a BBB-permeable peptide is used to increase the BBB permeability as an effective strategy for the treatment of CNS inflammatory diseases (Lim et al. 2015). Recently, a neuronal damage inhibitor was linked to the cell-penetrating Tat peptide for modulating the BBB adherence, thereby promoting the neuronal uptake and permeation of this inhibitor across the BBB (Zhan et al. 2011). Our finding indicated that peptides, such as FD7 and CCD, and Afa-loaded nanoparticles, including Afa/LPN, Afa/LPN-FD7, and Afa/LPN-CCD, did not cause a significant cytotoxic effect on bEnd.3 cells (Fig. 4). Afa/LPN-FD7 and Afa/ LPN-CCD across the BBB model of bEnd. 3 cells enhanced the cytotoxic effect of Afa on PC9 cells. FD7 and CCD perturbed TJ proteins, such as claudin 5 and ZO-1 (Fig. 8A), decreased the TEER (Fig. 7C) of bEnd.3 cells, and increased the permeability (\%) of FITC-dextran across the bEnd.3 cells (Fig. 7D). This result suggested that these formulations might cross the BBB via paracellular pathway by disrupting the interactions of $\mathrm{TJ}$ proteins, diminishing the $\mathrm{TJ}$-associated barrier functions without affecting the protein expression of TJs (Fig. 8B). Furthermore, Afa/LPN-FD7 and Afa/LPN-CCD were partially transported across the BBB via transcellular pathway by clathrin- and caveolaemediated transcytosis (Fig. 7AB). These findings revealed the effective activation of both paracellular and transcellular pathways by these LPN modified with TJ-interrupted peptides to facilitate Afa delivery across the BBB for promoting the cytotoxic effect on PC9 cells (Fig. 6B). Afa/LPN-CCD exhibited more negative zeta potential, possibly due to two carboxylate groups in the side chains of $\mathrm{CCD}$, thus displaying more repulsion and smaller particle size than that of Afa/LPN-FD7 (Table 1). CCD, a cyclic peptide from human E-cadherin (Laksitorini et al. 2015), might condense itself into compact structure to generate pores in the intercellular junctions of the BBB (Alaofi et al. 2017) and thus demonstrated stronger perturbing effect on the $\mathrm{BBB}$ integrity and function than that FD7. Hence, Afa/LPN-CCD showed more enhancement on cytotoxicity of Afa against PC9 cells than that of Afa/LPN-FD7 (Fig. 6B). The schematic of the transport of Afa/ LPN-FD7 and Afa/LPN-CCD across the BBB model of bEnd.3 cells via paracellular and transcellular pathways to release Afa is shown in Fig. 9.

\section{Conclusions}

Collectively, Afa encapsulated in LPN coated with TJ-modulating FD7 or CCD can improve the cytotoxicity of Afa across the $\mathrm{BBB}$ on $\mathrm{PC} 9$ cells via paracellular and transcellular pathways. TJ-modulating peptide-modified LPN can be a potential platform for the delivery of chemotherapeutics across the BBB to the brain for the treatment of brain tumors or the BM of tumors such as NSCLC. 


\section{Materials and methods}

\section{Materials}

Afa was obtained from MedChem Express, Monmouth, NJ, USA. FD7 and CCD were purchased from Kelowna Biotech (Taipei, Taiwan) at $>95 \%$ purity. Lecithin was purchased from Alfa Aesar (Heysham, LNH, Kingdom) and DSPE-PEG2000-NHS was obtained from Nanocs Inc. (Boston, MA, USA). All cell culture medium and reagents were bought from Promega (Madison, WI, USA), Invitrogen (Carlsbad, CA, USA), Gibco BRL (Grand Island, NY, USA), or Hyclone (Logan, UT, USA). All other chemical reagents were obtained from either Merck (Darmstadt, Germany) or Sigma-Aldrich.

\section{Preparation of Afa/LPN and peptide-conjugated Afa/LPN}

Peptide-linked lipids were initially produced by dispersing FD7 or CCD peptides and DSPE-PEG2000-NHS at a molar ratio of 1:1 in chloroform and methanol (1:1) for $24 \mathrm{~h}$ at room temperature. After forming the peptide-lipid conjugates, the organic solvent was evaporated and the residue was then dialyzed against water by dialysis bag (molecular weight cutoff at $0.5-1 \mathrm{kDa}$; Spectrum Laboratories, CA, USA). The purified peptidelinked lipids were collected by freeze-drying. The conjugation has been confirmed using a MALDI-TOF mass spectrometer (MALDI-TOF MS).

Afa-loaded nanoparticles were prepared by an o/w emulsion method. mPEG-DSPE $(2 \mathrm{mg} / \mathrm{ml})$ and lecithin $(2 \mathrm{mg} / \mathrm{ml})$ were mixed into PBS buffer to prepare aqueous dispersion. To prepare $1.6 \mathrm{nM} \mathrm{Afa/LPN}, 6.4 \mu \mathrm{l}$ of $1 \mu \mathrm{M}$ afatinib in DMSO was added to $4 \mathrm{ml}$ PLGA (2 mg/ml; 50:50 LA:GA; MW 35,000-45,000) in acetone solution. Afa in PLGA solution was then cautiously added drop by drop into aqueous lipid dispersion. The

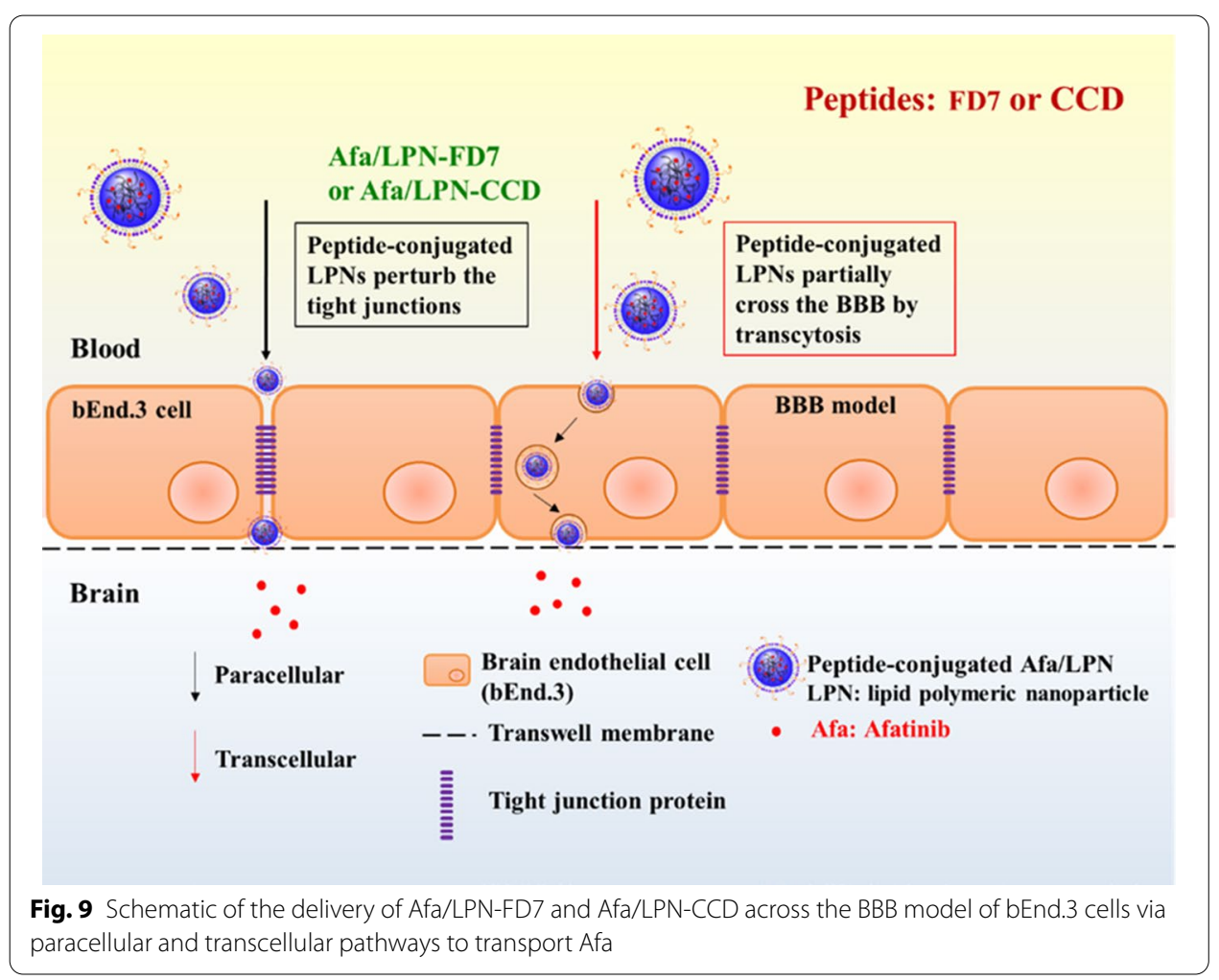


resulting o/w emulsion was sonicated for 10 min using a bath sonicator at $50 \%$ amplitude. The organic solvent was then removed by constant evaporation under vacuum at room temperature. The preparation of Afa/LPN-FD7 and Afa/LPN-CCD was the same as the preparation of Afa/LPN, but mPEG2000-DSPE was replaced with the individual peptide-conjugated lipid.

\section{Characterization of Afa-loaded nanoparticles}

Afa-contained nanoparticles dispersion was centrifuged at $25,200 \mathrm{~g}$ and $4{ }^{\circ} \mathrm{C}$ through an ultracentrifuge filter $\left(\right.$ Amicon $^{\circledR}, \mathrm{MW}: 10 \mathrm{kDa}$ ). Afa in the supernatant was detected by HPLC. The HPLC system consists of a pump (PM1110; Hitachi, Tokyo, Japan), an autosampler (Primaide 1210; Hitachi), a reverse C18 column (Phenomenex), and a L2420 UV detector (Hitachi). The mobile phase was prepared by mixing water, acetonitrile, and methanol $(55: 25: 20 \mathrm{v} / \mathrm{v})$. The solution was degassed by a sonicator before detection. The UV detection was carried out at wavelength $254 \mathrm{~nm}$ and flow rate was set at $1.0 \mathrm{~mL} / \mathrm{min}$. Records were analyzed from three independent experiments. Encapsulation efficiency (EE \%) was computated by the following formula:

$$
E E \%=\left[\left(W_{e}-W_{s}\right) / W_{e}\right] \times 100 \%,
$$

where We is the weight of added Afa and Ws is the weight of Afa in the supernatant.

The size distribution and zeta potential of nanoparticles were monitored by Zetasizer Nano ZS90 dynamic light scattering system (Malvern Instruments Ltd., Malvern, Worcestershire, UK) at $25^{\circ} \mathrm{C}$ and a detection angle of $90^{\circ}$ for LPN dispersion. The resulting particle size distribution was plotted as the intensity (\%) of LPN versus size in diameter $(\mathrm{nm})$. Data were analyzed from four individual measurements and computed by a cumulant method to automatically obtain polydispersity index using Zetasizer family software v7.11.

The morphology of nanoparticles was captured under transmission electron microscopy (JEM-2000EX, JEOL, Ltd., Tokyo, Japan). The samples were diluted with doubledistilled water and dropped on the grid for $1 \mathrm{~min}$. The samples were immersed in 1\% phosphotungstic acid. The excess of solution was sucked from the edge with a filter paper and then dried in the air at room temperature.

\section{In vitro release of Afa from nanoparticles}

The release study of Afa from Afa dispersion, Afa/LPN, Afa/LPN-FD7, and Afa/LPNCCD was implemented by dialysis method. $1 \mathrm{~mL}$ of Afa-contained formulation at Afa equivalent concentration of $10 \mu \mathrm{M}$ was suspended into a dialysis bag $(3.5 \sim 5 \mathrm{kDa}$ MWCO membrane, Spectrum, Inc., Rancho Dominguez, CA, USA). The dialysis medium was $100 \mathrm{~mL}$ PBS $(\mathrm{pH}=7.4)$ with stirring at $18 \mathrm{~g}$ and $37^{\circ} \mathrm{C}$. At time points of 0.5 , $1,2,4,8,12,24$, and $48 \mathrm{~h}, 0.1 \mathrm{~mL}$ sample was removed from the dialyzer and the same volume of PBS was supplemented into the dialyzer to maintain the same volume of solution. Afa concentration in each sample was analyzed by HPLC. The cumulative release of Afa was then computated. 


\section{Culture of PC-9 cells and bEnd. 3 cells and cytotoxicity evaluation by SRB assay}

bEnd. 3 cells were grown in Dulbecco's Modified Eagle Medium (DMEM) nourished with F-12 (DMEM/F12; Gibco), 10\% fetal bovine serum (FBS), and 1\% penicillin/streptomycin. PC9 cells are human lung adenocarcinoma cells with EGFR exon 19 deletion. Cells were grown in Roswell Park Memorial Institute-1640 Medium (RPMI-1640; Hyclone) supplemented with $10 \% \mathrm{FBS}$ and $1 \%$ penicillin/streptomycin. Cell lines were all maintained in the incubator with $5 \% \mathrm{CO}_{2}$ at $37{ }^{\circ} \mathrm{C}$. Other cell culture reagents were puchased from Corning. bEnd. 3 and PC9 cells were seeded at density of $2 \times 10^{4}$ cells/ well and $4 \times 10^{3}$ cells/well in 96-well flat transparent plates and cultured for $24 \mathrm{~h}$. Different concentrations of Afa, FD7, or CCD in free or the corresponding LPN formulations were added to the medium and cultured for $48 \mathrm{~h}$, as indicated in Figs. 4 and 6 . The cultured medium was then removed and the cytotoxicity was determined by SRB assay. Briefly, after adding $0.04 \%$ SRB into each well for $10 \mathrm{~min}$, the individual well was washed three times with $1 \%$ acetic acid. After drying the wells at room temperature for $24 \mathrm{~h}, 10 \mathrm{mM}$ Tris base was added to the separate well. The absorbance was measured at $540 \mathrm{~nm}$ by a Tecan microplate reader.

\section{Establishment of in vitro BBB model: morphology of barrier integrity}

bEnd. 3 cells were seeded at a density of $2 \times 10^{4}$ cells/insert on ThinCert ${ }^{\mathrm{TM}}$ cell culture inserts (Greiner Bio-One, Germany). Cells were cultured up to 6 days and the intergriy of monolayers were examined to verify if the BBB model was suitable for the following experiments. The bEnd.3 cells were stained with SRB and morphology of the BBB model was detected. The images of mophology was observed under a Nikon Diaphot 300 Inverted Tissue Culture Microscope (Nikon, Tokyo, Japan).

\section{Verification of BBB model: TEER measurement}

bEnd. 3 cells were seeded at a density of $2 \times 10^{4}$ cells/insert on ThinCert ${ }^{\mathrm{TM}}$ cell culture inserts. After culture up to 6 days, the monolayers were formed for the following experiments as a BBB model. Two electrodes of Millicell ERS-2 Epithelial Volt-Ohm Meter (Millipore, Billerica, MA, USA) were mounted in the medium of the upper insert and lower well, respectively. The electrodes can detect the resistance values across the inserts with or without the bEnd.3 monolayers. TEER was calculated by the following formula:

$$
T E E R=\left(R_{\text {total }}-R_{\text {blank }}\right) \times A\left(\Omega \times \mathrm{cm}^{2}\right)
$$

where $R_{\text {total }}$ is the resistance acorss the cell layers on permeable inserts, $R_{\text {blank }}$ is the resistance of peremable inserts without cells and A is the surface area of insert membrane $\left(0.336 \mathrm{~cm}^{2}\right)$.

\section{Verification of BBB model: permeability measurement}

After incubation, the lower wells were replaced with $0.6 \mathrm{~mL}$ HBSS buffer. $0.1 \mathrm{~mL}$ FITCdextran $(0.09 \mathrm{mg} / \mathrm{mL}$; MW 70,000; a paracellular marker) were then added to the upper inserts. After incubation of these cells in the dark at $37{ }^{\circ} \mathrm{C}$ for $1 \mathrm{~h}, 0.1 \mathrm{~mL}$ solution of 
lower well was collected. Fluorescence intensity of FITC-dextran was measured at excitation $494 \mathrm{~nm}$ and emission at $521 \mathrm{~nm}$ using a Tecan Infinite $200^{\circledR}$ PRO multimode microplate reader (Männedorf, Switzerland). The relative percentage of permeability was calculated by normalizing the blank values (without cell seeding on the inserts; Fig. 5D) or CTR (no treatment; Fig. 7D) as 100\%.

\section{Cytotoxicity of Afa-formulations across the BBB on PC9 cells by SRB assay}

bEnd. 3 cells were seeded at a density of $2 \times 10^{4}$ cells/inser on the upper chamber of ThinCert $^{\mathrm{TM}} 24$-well inserts. PC9 cells were seed at a density of $2 \times 10^{4}$ cells/well on the lower plates of 24 well and cultured for $24 \mathrm{~h}$. bEnd. 3 cells were cultured for 5 days and treatments of different Afa-formulations were added into the medium in the upper chamber. After treatments of these cells for $48 \mathrm{~h}$, the upper inserts were removed. The cytotoxicity was examined by SRB assay. Cell viability \% was normalized relatively to the control by dividing the absorbance values of the treated cells with that of cells of medium control.

\section{Western blot assay}

bEnd. 3 and PC9 cells $\left(2 \times 10^{5}\right.$ cells/dish) were incubated in the $6 \mathrm{~cm}$ dish, and different Afa-formulations were added and cultured for $48 \mathrm{~h}$. After lysis of cells by radioimmunoprecipitation assay (RIPA) buffer (Cell Signaling, Beverly, MA, USA), the proteins were extracted and quantified by BCA Protein Assay Kit (Thermo Fisher Scientific, Waltham, MA, USA). Proteins (30 $\mu \mathrm{g} / \mathrm{lane})$ were run by $8-13.5 \%$ sodium dodecyl sulfate-polyacrylamide gel electrophoresis and moved to a polyvinylidene difluoride membrane at $80 \mathrm{~V}$ for $120 \mathrm{~min}$. After blocking, the membranes were incubated with primary antibodies against Bax, Bcl-2, Casp-3, RIP-1, RIP-3, Claudin 5, ZO-5, E-cadherin, and B-actin overnight at $4{ }^{\circ} \mathrm{C}$. Then, the samples were immersed with horseradish peroxidase-conjugated secondary IgG at room temperature for $1 \mathrm{~h}$. The relative protein expression was examined using a chemiluminescent ImageQuant LAS 4000 Scanner of GE Healthcare (Piscataway, NJ, USA).

\section{Endocytic uptake mechanisms of Rh123-loaded nanoparticles}

bEnd. 3 cells were seed in the 24-well plate overnight and pretreated with different endocytosis inhibitors, including chlorpromazine hydrochloride (CPZ; $10 \mu \mathrm{M}$, clathrin-mediated endocytosis inhibitor), 5-(N,N-dimethyl) amiloride hydrochloride (DMA; $10 \mu \mathrm{g} /$ $\mathrm{mL}$, micropinocytosis inhibitor), nystatin (Nys; $20 \mu \mathrm{g} / \mathrm{mL}$, and caveolae-mediated endocytosis inhibitor) for $30 \mathrm{~min}$, and then treated with Rh123-loaded nanoparticles for $3 \mathrm{~h}$. Rh123 is a cell-permeant dye with green fluorescence $(\mathrm{Ex} / \mathrm{Em}=488 / 534 \mathrm{~nm})$ (Petrat et al. 2003). It is used as a fluorescent probe of Afa for incorporation into nanoparticles without cytotoxic effects. After treatments, the cells were collected, centrifuged at $300 \mathrm{~g}$ and $4{ }^{\circ} \mathrm{C}$ and re-suspended in cold PBS. The harvested cells were monitored by FACSCalibur flow cytometer (BD Biosciences, San Jose, CA, USA). Fluorescence was collected through a FL1 filter $(515-545 \mathrm{~nm})$ for Rh123 and fluorescence signals were transferred into a logarithmic scale. Data processing and analysis were conducted by BD FACStation $^{\text {TM }}$ software (BD Biosciences). Each group was carried out in triplicate. 


\title{
Effect of Afa/nanoparticles on immunofluorescence staining of TJ proteins by confocal laser scanning microscope (CLSM)
}

Immunostaining was performed for TJ proteins, including claudin-5 and ZO-1. After treatments, cells were washed with phosphate buffer (PBS), and fixed with mixture of ice cold acetone and methanol for $10 \mathrm{~min}$ at room temperature. After blocking with $3 \%$ bovine serum albumin in PBS, cells were incubated with primary antibodies of mouse anti-claudin-5 (Invitrogen, Camarillo, CA, USA) and rabbit anti-ZO-1 (Invitrogen, Camarillo, CA, USA) overnight. The cells were detected with secondary antibodies of FITC-labeled anti-rabbit (Jackson Immunoresearch, West Grove, PA, USA) and Texas red-labeled anti-mouse (Jackson Immunoresearch, West Grove, PA, USA) for $1 \mathrm{~h}$. DAPI was used to stain cell nuclei. After mounting the samples, these images were visualized by a confocal laser scanning microscope (Olympus FV10i, Olympus America Inc., Center Valley, PA, USA).

\section{Statistical analysis}

Experimental data were expressed as the mean $\pm \mathrm{SD}$ and analyzed by Student's $t$-test. $p<0.05$ was considered statistically significant.

\begin{abstract}
Abbreviations
NSCLC: Non-small-cell lung cancer; EGFR: Epidermal growth factor receptor; CNS: Central nervous system; BM: Brain metastases; LM: Leptomeningeal metastases; EGFR-TKIs: Epidermal growth factor receptor-tyrosine kinase inhibitors; BBB: Epidermal blood-brain barrier; TJs: Tight junctions; HER: Human epidermal growth factor receptor; Afa: Afatinib; ErbB: Pan-erythroblastic leukemia viral oncogene homolog; CSF: Cerebral spinal fluid; LPN: Lipid polymeric nanoparticle; PLGA: Polylactic-co-glycolic acid; CCD: Cyclic (CD) peptide; Afa/LPN-FD7: Afa-loaded LPN coated with FD7; Afa/LPN-CCD: Afaloaded LPN coated with CCD; bEnd.3 cells: Brain-derived endothelial cells.3; PC9: Human lung adenocarcinoma PC9 cells; TEM: Transmission electron microscopy; EE\%: Encapsulation efficiency; Pdl: Polydispersity index; SRB: Sulforhodamine B; TEER: Transendothelial electrical resistance; FBS: Fetal bovine serum; FITC-dextran: Fluorescein isothiocyanate-conjugated dextran; RIP: Kinase receptor-interacting protein; Rh123: Rhodamine 123; DMA: 5-(N,N-Dimethyl) amiloride; CPZ: Chlorpromazine; CLSM: Confocal laser scanning microscopy; ZO-1: Zonula occludens-1; CTR: Control.
\end{abstract}

\section{Supplementary Information}

The online version contains supplementary material available at https://doi.org/10.1186/s12645-021-00084-w.

Additional file 1: Figure S1. Stability of afatinib (Afa) in LPN-FD7 and LPN-CCD for 28 days. Afa/LPN-FD7 and Afa/ LPN-CCD were stored at $4{ }^{\circ} \mathrm{C}$ and PBS of pH7.4 for 28 days. The size was measured by Zetasizer. The values are the mean \pm standard error (SD). For each group, $n=3$.

\section{Acknowledgements}

We thank Professor Chuen-Mao Yang (Program for Biotech Pharmaceutical Industry, China Medical University) for kindly providing the bEnd. 3 cells.

\section{Authors' contributions}

YL conceived, designed and interpreted the experiments as well as wrote the original and revised manuscript; SH, CC, and CW performed the experiments and analyzed the data; $\mathrm{HL}$ and $\mathrm{AML}$ conceived and interpreted the experiments; $\mathrm{YL}$, $\mathrm{HL}$, and $\mathrm{AML}$ contributed reagents/materials/analysis tools. All authors read and approved the final manuscript.

\section{Funding}

This research was funded by National Yang Ming Chiao Tung University and Cheng Hsin General Hospital (CY10935; CY1 1004), Ministry of Science and Technology of Taiwan (MOST 107-2320-B-010-015-MY3; MOST 106-2320-B-010-005), Veterans General Hospitals and University System of Taiwan Joint Research Program (VGHUST110-G2-2-2; VGHUST109-

V7-3-2), and Center for Advanced Pharmaceutics and Drug Delivery Research, National Yang Ming Chiao Tung University.

Availability of data and materials

All data and materials are included in the manuscript. 


\section{Declarations}

Ethics approval and consent to participate

Not applicable.

\section{Consent for publication}

Not applicable.

\section{Competing interest}

The authors declare no conflict of interest. The funders had no involvement in the design of the study; in the collection, analyses, or interpretation of data; in the writing of the manuscript, or in the decision to publish the results.

\section{Author details}

${ }^{1}$ Institute of Pharmacology, National Yang Ming Chiao Tung University, Taipei 112, Taiwan. ${ }^{2}$ Faculty of Pharmacy, National Yang Ming Chiao Tung University, Taipei 112, Taiwan. ${ }^{3}$ Center for Advanced Pharmaceutics and Drug Delivery Research, National Yang Ming Chiao Tung University, Taipei 112, Taiwan. ${ }^{4}$ Division of Colorectal Surgery, Surgical Department, Chen-Hsin General Hospital, Taipei, Taiwan. ${ }^{5}$ Department of Healthcare Information and Management, Ming Chuan University, Taoyuan, Taiwan. ${ }^{6}$ Department of Medical Research, Taipei Veterans General Hospital, Taipei 112, Taiwan.

Received: 6 February 2021 Accepted: 19 May 2021

Published online: 07 June 2021

\section{References}

Abdallah SM, Wong A. Brain metastases in non-small-cell lung cancer: are tyrosine kinase inhibitors and checkpoint inhibitors now viable options? Curr Oncol. 2018;25:S103-s114.

Alaofi A, On N, Kiptoo P, Williams TD, Miller DW, Siahaan TJ. Comparison of linear and cyclic His-Ala-Val peptides in modulating the blood-brain barrier permeability: impact on delivery of molecules to the brain. J Pharm Sci. 2016;105:797-807.

Alaofi A, Farokhi E, Prasasty VD, Anbanandam A, Kuczera K, Siahaan TJ. Probing the interaction between cHAVc3 peptide and the EC1 domain of E-cadherin using NMR and molecular dynamics simulations. J Biomol Struct Dyn. 2017;35:92-104

Bocsik A, Walter FR, Gyebrovszki A, Fulop L, Blasig I, Dabrowski S, Otvos F, Toth A, Rakhely G, Veszelka S, Vastag M, SzaboRevesz P, Deli MA. Reversible opening of intercellular junctions of intestinal epithelial and brain endothelial cells with tight junction modulator peptides. J Pharm Sci. 2016;105:754-65.

Chen Y, Liu L. Modern methods for delivery of drugs across the blood-brain barrier. Adv Drug Deliv Rev. 2012;64:640-65.

D'Arcangelo M, Hirsch FR. Clinical and comparative utility of afatinib in non-small cell lung cancer. Biologics. 2014;8:183-92.

Deli MA. Potential use of tight junction modulators to reversibly open membranous barriers and improve drug delivery. Biochim Biophys Acta. 2009;1788:892-910.

Dempke WC, Edvardsen K, Lu S, Reinmuth N, Reck M, Inoue A. Brain metastases in NSCLC - are TKls changing the treatment strategy? Anticancer Res. 2015;35:5797-806.

Fidler IJ. The biology of brain metastasis: challenges for therapy. Cancer J. 2015;21:284-93.

Herman RE, Makienko EG, Prieve MG, Fuller M, Houston ME Jr, Johnson PH. Phage display screening of epithelial cell monolayers treated with EGTA: identification of peptide FDFWITP that modulates tight junction activity. J Biomol Screen. 2007;12:1092-101.

Hirsh V. Next-generation covalent irreversible kinase inhibitors in NSCLC: Focus on afatinib. BioDrugs. 2015;29:167-83.

Hoffknecht P, Tufman A, Wehler T, Pelzer T, Wiewrodt R, Schutz M, Serke M, Stohlmacher-Williams J, Marten A, Maria Huber R, Dickgreber NJ. Efficacy of the irreversible ErbB family blocker afatinib in epidermal growth factor receptor (EGFR) tyrosine kinase inhibitor (TKI)-pretreated non-small-cell lung cancer patients with brain metastases or leptomeningeal disease. J Thorac Oncol. 2015;10:156-63.

Hong ST, Lin H, Wang CS, Chang CH, Lin AM, Yang JC, Lo YL. Improving the anticancer effect of afatinib and microRNA by using lipid polymeric nanoparticles conjugated with dual pH-responsive and targeting peptides. J Nanobiotechnol. 2019;17:89.

Hurvitz SA, Shatsky R, Harbeck N. Afatinib in the treatment of breast cancer. Expert Opin Investig Drugs. 2014;23:1039-47.

Laksitorini MD, Kiptoo PK, On NH, Thliveris JA, Miller DW, Siahaan TJ. Modulation of intercellular junctions by cyclic-ADT peptides as a method to reversibly increase blood-brain barrier permeability. J Pharm Sci. 2015;104:1065-75.

Lee CC, Shiao HY, Wang WC, Hsieh HP. Small-molecule EGFR tyrosine kinase inhibitors for the treatment of cancer. Expert Opin Investig Drugs. 2014;23:1333-48.

Li SH, Hsieh MH, Fang YF. Afatinib in treatment-naive patients With EGFR-mutated lung adenocarcinoma with brain metastasis: a case series. Medicine (baltimore). 2015;94:e1739.

Li D, Ambrogio L, Shimamura T, Kubo S, Takahashi M, Chirieac LR, Padera RF, Shapiro Gl, Baum A, Himmelsbach F, Rettig WJ, Meyerson M, Solca F, Greulich H, Wong KK. BIBW2992, an irreversible EGFR/HER2 inhibitor highly effective in preclinical lung cancer models. Oncogene. 2008;27:4702-11.

Li G, Simon MJ, Cancel LM, Shi ZD, Ji X, Tarbell JM, Morrison B 3rd, Fu BM. Permeability of endothelial and astrocyte cocultures: in vitro blood-brain barrier models for drug delivery studies. Ann Biomed Eng. 2010;38:2499-511.

Lim S, Kim WJ, Kim YH, Lee S, Koo JH, Lee JA, Yoon H, Kim DH, Park HJ, Kim HM, Lee HG, Yun Kim J, Lee JU, Hun Shin J, Kyun Kim L, Doh J, Kim H, Lee SK, Bothwell AL, Suh M, Choi JM. dNP2 is a blood-brain barrier-permeable peptide enabling ctCTLA-4 protein delivery to ameliorate experimental autoimmune encephalomyelitis. Nat Commun. $2015 ; 6: 8244$ 
Lin KH, Hong ST, Wang HT, Lo YL, Lin AM, Yang JC. Enhancing anticancer effect of gefitinib across the blood-brain barrier model using liposomes modified with one alpha-helical cell-penetrating peptide or glutathione and tween 80. Int J Mol Sci. 2016;17:E1998.

Liu CY, Wang CL, Li SH, Hsu PC, Chen CH, Lin TY, Kuo CH, Fang YF, Ko HW, Yu CT, Yang TY, Yang CT. The efficacy of 40 mg versus dose de-escalation to less than $40 \mathrm{mg}$ of afatinib (Giotrif) as the first-line therapy for patients with primary lung adenocarcinoma harboring favorable epidermal growth factor mutations. Oncotarget. 2017;8:97602-12.

Mandal B, Mittal NK, Balabathula P, Thoma LA, Wood GC. Development and in vitro evaluation of core-shell type lipid-polymer hybrid nanoparticles for the delivery of erlotinib in non-small cell lung cancer. Eur J Pharm Sci. 2016;81:162-71.

Masserini M. Nanoparticles for brain drug delivery. ISRN Biochemistry. 2013;2013:18.

On NH, Miller DW. Transporter-based delivery of anticancer drugs to the brain: improving brain penetration by minimizing drug efflux at the blood-brain barrier. Curr Pharm Des. 2014;20:1499-509.

Petrat F, Pindiur S, Kirsch M, de Groot H. "Mitochondrial" photochemical drugs do not release toxic amounts of 1O(2) within the mitochondrial matrix space. Arch Biochem Biophys. 2003;412:207-15.

Rijcken CJ, Veldhuis TF, Ramzi A, Meeldijk JD, van Nostrum CF, Hennink WE. Novel fast degradable thermosensitive polymeric micelles based on PEG-block-poly(N-(2-hydroxyethyl)methacrylamide-oligolactates). Biomacromol. 2005;6:2343-51.

Rijcken CJ, Snel CJ, Schiffelers RM, van Nostrum CF, Hennink WE. Hydrolysable core-crosslinked thermosensitive polymeric micelles: synthesis, characterisation and in vivo studies. Biomaterials. 2007;28:5581-93.

Sasikala AR, GhavamiNejad A, Unnithan AR, Thomas RG, Moon M, Jeong YY, Park CH, Kim CS. A smart magnetic nanoplatform for synergistic anticancer therapy: manoeuvring mussel-inspired functional magnetic nanoparticles for $\mathrm{pH}$ responsive anticancer drug delivery and hyperthermia. Nanoscale. 2015;7:18119-28.

Sim TM, Tarini D, Dheen ST, Bay BH, Srinivasan DK. Nanoparticle-based technology approaches to the management of neurological disorders. Int J Mol Sci. 2020;21:6070-101.

Trabulo S, Resina S, Simoes S, Lebleu B, Pedroso de LMC. . A non-covalent strategy combining cationic lipids and CPPs to enhance the delivery of splice correcting oligonucleotides. J Control Release. 2010;145:149-58.

Trabulo S, Cardoso AL, Cardoso AM, Morais CM, Jurado AS, Pedroso de LMC. . Cell-penetrating peptides as nucleic acid delivery systems: from biophysics to biological applications. Curr Pharm Des. 2013;19:2895-923.

Wei YF, Lim CK, Tsai MS, Huang MS, Chen KY. Intracranial responses to afatinib at different doses in patients with EGFRmutated non-small-cell lung carcinoma and brain metastases. Clin Lung Cancer. 2019;20:e274-83.

Yang JC, Wu YL, Schuler M, Sebastian M, Popat S, Yamamoto N, Zhou C, Hu CP, O'Byrne K, Feng J, Lu S, Huang Y, Geater SL, Lee KY, Tsai CM, Gorbunova V, Hirsh V, Bennouna J, Orlov S, Mok T, Boyer M, Su WC, Lee KH, Kato T, Massey D, Shahidi M, Zazulina V, Sequist LV. Afatinib versus cisplatin-based chemotherapy for EGFR mutation-positive lung adenocarcinoma (LUX-Lung 3 and LUX-Lung 6): analysis of overall survival data from two randomised, phase 3 trials. Lancet Oncol. 2015;16:141-51.

Yang JC, Sequist LV, Zhou C, Schuler M, Geater SL, Mok T, Hu CP, Yamamoto N, Feng J, O'Byrne K, Lu S, Hirsh V, Huang Y, Sebastian M, Okamoto I, Dickgreber N, Shah R, Marten A, Massey D, Wind S, Wu YL. Effect of dose adjustment on the safety and efficacy of afatinib for EGFR mutation-positive lung adenocarcinoma: post hoc analyses of the randomized LUX-Lung 3 and 6 trials. Ann Oncol. 2016;27:2103-10.

Zeng Q, Wang J, Cheng Z, Chen K, Johnstrom P, Varnas K, Li DY, Yang ZF, Zhang X. Discovery and evaluation of clinical candidate AZD3759, a potent, oral active, central nervous system-penetrant, epidermal growth factor receptor tyrosine kinase inhibitor (EGFR TKI). J Med Chem. 2015;58:8200-15.

Zhan F, Chen W, Wang Z, Lu W, Cheng R, Deng C, Meng F, Liu H, Zhong Z. Acid-activatable prodrug nanogels for efficient intracellular doxorubicin release. Biomacromol. 2011;12:3612-20.

\section{Publisher's Note}

Springer Nature remains neutral with regard to jurisdictional claims in published maps and institutional affiliations. 